\title{
INFLUÊNCIA DO USO DA IRRIGAÇÃO DURANTE O ACABAMENTO E POLIMENTO DE RESINAS COMPOSTAS: RUGOSIDADE SUPERFICIAL, ESTABILIDADE DE COR E MORFOLOGIA DE SUPERFÍCIE
}

\author{
INFLUENCE OF WET FINISHING AND POLISHING ON COMPOSITE RESINS: \\ SURFACE ROUGHNESS, COLOR STABILITY AND SURFACE MORPHOLOGY
}

\author{
Marcus Vinícius Nadler Mendonça dos Reis Perillo de FREITAS ${ }^{1}$; Denise Tavares Nadler Perillo de FREITAS²; Letícia Nunes de \\ ALMEIDA3; Ana Paula Rodrigues de MAGALHÃES4; Paula de Carvalho Cardoso5; Rafael de Almeida DECURCIO 6 \\ 1 - Especialista em Dentística Restauradora, professor do Curso de Especialização em Dentistíca pela ABO/Goiás \\ 2 - Aluna do Curso de Especialização em Dentistíca Restauradora pela ABO/Goiás \\ 3 - Doutoranda em Odontologia pela Universidade Federal de Goiás, professora do curso de especialização em Dentística Restauradora pela ABO/Goiás \\ 4 - Doutora em Dentística Restauradora pela Universidade De São Paulo, professora do Curso de Especialização em Dentística pela ABO/Goiás \\ 5 - Doutora em Dentística Restauradora pela Universidade Federal de Santa Catarina, professora do Curso de Especialização em Dentística pela \\ ABO/Goiás \\ 6 - Mestre em Reabilitação Oral Universidade Federal de Uberlândia, Professor do Curso de Especialização em Dentística da ABO/Goiás
}

\section{RESUMO}

Objetivo: Avaliar rugosidade superficial (RS), estabilidade de cor (EC) e morfologia de superfície (MS) de um compósito nanoparticulado após diferentes protocolos de acabamento/polimento com ou sem irrigação. Material e métodos: Cem espécimes foram confeccionados e divididos em 10 grupos $(n=10)$ conforme protocolo de acabamento/polimento: Tira de poliéster; discos abrasivos (DA); DA+espirais (DAE); DA+multi-laminada+espirais (DAME); espirais (E); e nenhum polimento. Protocolos com DA, DAE, DAME, e E foram realizados a seco e com irrigação. Após acabamento/polimento, os espécimes foram armazenados em água destilada à $37^{\circ} \mathrm{C}$. Durante 14 dias, cinco espécimes de cada grupo foram imersos em café por $5 \mathrm{~min} /$ dia. A EC foi analisada com Espectrofotômetro (CIEL*a*b*) 0, 7 e 14 dias após armazenamento em água ou imersão em café. A RS foi analisada com Rugosímetro (parâmetro Ra) e MS foi analisada com Microscopia Eletrônica de Varredura (MEV). ANOVA, Tukey e Teste $\mathrm{T}$ foram realizados para comparações entre os grupos $(\alpha=0,05)$. Resultados: A irrigação promoveu maior EC nos protocolos DAE e E. Os diferentes protocolos promoveram variação de cor semelhante após 14 dias de armazenamento em água. O protocolo $\mathrm{E}$ a seco promoveu os maiores valores RS, sendo inferior ao grupo DAME, que promoveu menor rugosidade e foi semelhante aos demais protocolos. Para DAME e E a irrigação foi significativa para diminuir a rugosidade. Na análise por MEV, E com irrigação apresentaram maior lisura superficial. Conclusão: A irrigação durante acabamento/polimento influencia na EC, RS e MS de compósitos. Os protocolos de acabamento/ polimento DAME e E com irrigação foram mais efetivos.

Palavras-chave: técnicas de polimento; rugosidade de superfície; variação de cor; resinas nanoparticuladas.

\section{INTRODUÇÃO}

Na saúde, a Odontologia vem acompanhando os anseios e a imensa demanda social por estética, aprimorando métodos, técnicas, equipamentos e materiais restauradores disponíveis. No quesito material, apesar de a cerâmica ser amplamente utilizada como material restaurador estético, as resinas compostas apresentam-se como uma excelente alternativa para o tratamento, oferecendo vantagens de menor custo, supressão de etapa laboratorial, reversibilidade técnica, conservadorismo e durabilidade ${ }^{1}$.

Com os avanços relacionados ao desempenho das resinas compostas, as resinas nanoparticuladas têm se destacado principalmente pelo tamanho reduzido das partículas de carga, melhorando as propriedades ópticas sem causar prejuízos em sua resistência ${ }^{2}$. Estudos têm mostrado melhor capacidade de polimento, longevidade do brilho de superfície e bom desempenho clínico em acompanhamento de até $10 \operatorname{anos}^{3-5}$.

Em reabilitações com resina composta, o acabamento e polimento são etapas primordiais para alcançar maior naturalidade e estética ao resultado final, garantindo longevidade ao tratamento ${ }^{6}$ e satisfação do paciente. Estas etapas envolvem a definição de forma e contornos anatômicos, remoção de excessos, redução da rugosidade, reprodução de texturas de superfície, além de promover brilho e lisura semelhantes ao esmalte dental ${ }^{6-8}$. A lisura da superfície da restauração é essencial para minimizar a retenção de placa bacteriana, irritação gengival, e descoloração da superfície ao longo do tempo ${ }^{9-11}$. Mudanças de cor ao longo do tempo dependem de diversos fatores, tais como a resina composta, o polimento e a lisura da superfície ${ }^{11}$. 
Acabamento e polimento são influenciados pela técnica e pelos materiais utilizados ${ }^{7,8}$. Para definição de forma, contorno anatômico e remoção de excessos instrumentos como discos abrasivos e brocas multilaminadas estão disponíveis. Estudos têm demonstrado bom desempenho de discos flexíveis impregnados com óxido de alumínio ${ }^{7}$, principalmente por não causar deslocamento das partículas de carga. Os sistemas de acabamento e polimento disponíveis geralmente possuem uma ampla variedade de discos, escovas e borrachas ${ }^{10}$. Alguns sistemas foram inseridos no mercado com a intenção de reduzir a quantidade de passos e simplificar a etapa de acabamento e polimento, entretanto os resultados obtidos por esses materiais ainda são controvérsios ${ }^{8,12}$.

$\mathrm{Na}$ literatura, diversos estudos relacionados às técnicas e materiais de acabamento e polimento estão disponíveis, entretanto o polimento a seco ou com irrigação foi pouco estudado. Sabe-se que, acabamento e polimento, quando realizados a seco podem gerar calor excessivo e formar trincas, conforme mostrado por Kaminedi em $2014^{13}$. Além disso, os grânulos presentes nos materiais usados para acabamento e polimento podem ser impregnados à superfície da resina e o aquecimento pode degradar a interface particular de carga/matriz resinosa, resultando no deslocamento de partículas de carga e aumento da rugosidade da superfície ${ }^{14,15}$.

Devido a quantidade de materiais e técnicas disponíveis não há um consenso na literatura sobre qual o melhor protocolo de acabamento e polimento para cada tipo de resina composta. Além disso, poucos estudos abordaram o uso da irrigação e sua influência durante o acabamento e polimento. Diante disso, o objetivo deste trabalho foi avaliar a influência do protocolo de acabamento e polimento e do uso da irrigação na rugosidade superficial, estabilidade de cor e morfologia de superfície de uma resina nanoparticulada. A hipótese inicial era a de que o uso da irrigação promoveria menor variação de cor e rugosidade superficial independente do protocolo de acabamento e polimento utilizado. As hipóteses nulas eram: $\left(\mathrm{H}_{0} 1\right)$ de que o uso da irrigação não influenciaria a variação de cor, a rugosidade superficial e a morfologia de superfície; $\left(\mathrm{H}_{0} 2\right)$ o protocolo de acabamento e polimento utilizado não influenciaria nas propriedades supracitadas.

\section{MATERIAL E MÉTODOS}

\section{Obtenção dos espécimes}

A composição, fabricante e lote da resina composta e materiais de acabamento e polimento utilizados neste estudo estão dispostos na Tabela 1 .

Quatro grupos foram criados $(n=10)$ tendo como fator em estudo o protocolo de acabamento e polimento utilizado, sendo realizados com o uso ou não de irrigação durante o polimento, totalizando 8 grupos. Além disso foi determinado um grupo controle positivo (CP), em que o espécime foi fotoativado sob uma tira de poliéster, e um controle negativo $(\mathrm{CN})$ em que não foi usada tira de poliéster e não foi realizado nenhum tipo de acabamento e polimento; totalizando 10 grupos. Esses grupos estão descritos na Tabela 2.

A partir de um molde de silicone foram obtidos 100 espécimes em forma de disco ( $3 \mathrm{~mm}$ de diâmetro $\mathrm{x} 1 \mathrm{~mm}$ de espessura). A resina composta foi inserida no molde em um único incremento com auxílio de uma espátula de resina, e foi fotoativada por 20 segundos a $1000 \mathrm{~mW} / \mathrm{cm}^{2}$ (Valo Cordless, Ultradent, South Jordan, UT, EUA). Apenas para o grupo CP, uma tira de poliéster foi posicionada sobre a resina composta previamente a fotoativação. Para os demais grupos, após a inserção da resina no molde com espátula foi realizada a fotoativação e nenhuma cobertura sobre o espécime. Após a obtenção dos espécimes, todos foram armazenados em água destilada a $37^{\circ} \mathrm{C}$ por 24 horas.

Tabela 1 - Composição, fabricante e lote dos materiais utilizados.

\begin{tabular}{|c|c|c|c|}
\hline Material & Composição & Fabricante & Lote \\
\hline Resina composta Filtek Z-350 XT & $\begin{array}{l}\text { Matriz: Bis-GMA, TEGDMA, UDMA, Bis-EMA. Partículas de carga: sílica }(5-75 \mathrm{~nm}) \text {, } \\
\text { nanoaglomerados de silica e zircônia }(0.6-1.4 \mu \mathrm{m})\end{array}$ & 3M ESPE, St. Paul, MN, EUA & 1813700374 \\
\hline Discos abrasivos Sof-Lex Pop-On & 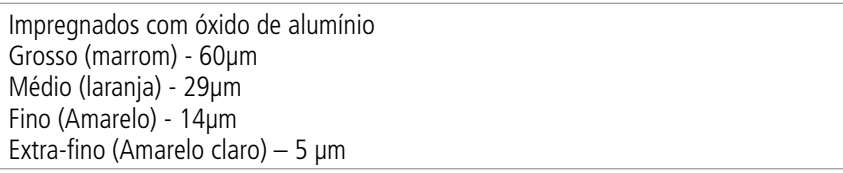 & 3M ESPE, St. Paul, MN, EUA & 1825500718 \\
\hline Sof-Lex discos espirais emborrachados & $\begin{array}{l}\text { Borracha abrasiva } \\
\text { Pré-polimento (bege) - grânulos óxido de alumínio } \\
\text { Polimento (rosa) - grânulos de óxido de alumínio }\end{array}$ & 3M ESPE, St. Paul, MN, EUA & 1825500718 \\
\hline Broca multilaminada (48L-010) & Broca carbide 12 lâminas & Angelus, Londrina, PR, Brasil & 1355 \\
\hline
\end{tabular}

Tabela 2 - Grupos experimentais de acordo com materiais e protocolos de polimento.

\begin{tabular}{|c|c|c|c|}
\hline Grupos & Materiais para polimento & Protocolo de polimento & Irrigação \\
\hline$C P$ & Tira de poliéster & - & - \\
\hline DA & Discos abrasivos & Discos Sof-Lex Pop-On (grosso, médio, fino e extra-fino) & Não \\
\hline DA-I & Discos abrasivos & Discos abrasivos (grosso, médio, fino e extra-fino) & $\operatorname{Sim}$ \\
\hline DAE & Discos abrasivos + espirais & Discos abrasivos (grosso e médio) + Sof-Lex espiral (bege e rosa) & Não \\
\hline DAE-I & Discos abrasivos + espirais & Discos abrasivos (grosso e médio) + Sof-Lex espiral (bege e rosa) & Sim \\
\hline DAME & Discos abrasivos + multilaminada + espirais & Discos abrasivos (grosso e médio) + broca multilaminada 12 lâminas + Sof-Lex espiral (bege e rosa) & Não \\
\hline DAME-I & Discos abrasivos + multilaminada + espirais & Discos (grosso e médio) + broca multilaminada 12 lâminas + Sof-Lex espiral (bege e rosa) & Sim \\
\hline E & Espirais & Sof-Lex espiral (bege e rosa) & Não \\
\hline $\mathrm{E}-\mathrm{I}$ & Espirais & Sof-Lex espiral (bege e rosa) & Sim \\
\hline $\mathrm{CN}$ & Sem polimento & - & - \\
\hline
\end{tabular}


Exceto para o grupo $\mathrm{CN}$, após o armazenamento, cada espécime foi polido pelo mesmo operador, treinado e calibrado, de acordo com as recomendações do fabricante para uso dos materiais. Cada disco (Sof-Lex Pop-On, 3M ESPE, EUA) e borracha abrasiva (Sof-Lex espiral, 3M ESPE, EUA) foi aplicado na superfície dos espécimes por 20 vezes em sentido horário com motor elétrico LB100 (Beltec Indústria Comércio Equipamentos, Araraquara, SP, Brasil) a $15.000 \mathrm{rpm}$. O peso do material de polimento sobre o espécime foi padronizado através de dispositivo específico criado para apoio do motor. A broca multilaminada 12 lâminas (48L-010, Angelus, Londrina, PR, Brasil) foi utilizada da mesma forma como descrito para os discos. Todos os materiais eram substituídos após uso, sendo utilizados sempre materiais novos para o polimento de cada espécime. Para os grupos polidos sob irrigação, um jato de água era aplicado constantemente durante o polimento por meio de bomba de água manual.

\section{Armazenamento}

Após a realização dos protocolos de acabamento e polimento os espécimes foram armazenadas em água destilada e foram mantidos à $37^{\circ} \mathrm{C}$. Durante 14 dias, cinco espécimes de cada grupo foram imersos em café (Café Rancheiro, Goiânia, GO, Brasil) por cinco minutos, 1 vez ao dia, retornando ao armazenamento em água destilada após a imersão em café. Dessa forma, dos 10 espécimes de cada grupo, cinco passaram pelo protocolo de imersão em café e cinco ficaram armazenados em água destilada, previamente aos testes.

\section{Rugosidade superficial}

A rugosidade superficial foi obtida por meio de Rugosímetro TR 210 (Digimess Instrumentos de Precisão Ltda., São Paulo, SP, Brasil) no parâmetro Ra (rugosidade média), dada em micrômetros com cut-off de $0,25 \mathrm{~mm}$. A rugosidade superficial foi aferida três vezes em cada amostra em posições distintas, após armazenamento em água ou café.

\section{Avaliação da cor}

Um operador treinado realizou a aferição da cor dos espécimes com espectrofotômetro (VITA Easyshade, Vita Zahnfabrik, Bad Säckingen, Alemanha), de acordo com o sistema CIEL*a*b* em 3 períodos: inicial (24 horas após a confecção das amostras), 7 dias após armazenamento em água destilada com ou sem imersão em café, 14 dias após armazenamento em água destilada com ou sem a imersão em café. A ponta do espectrofotômetro foi posicionada paralelamente sobre o espécime e as mensurações

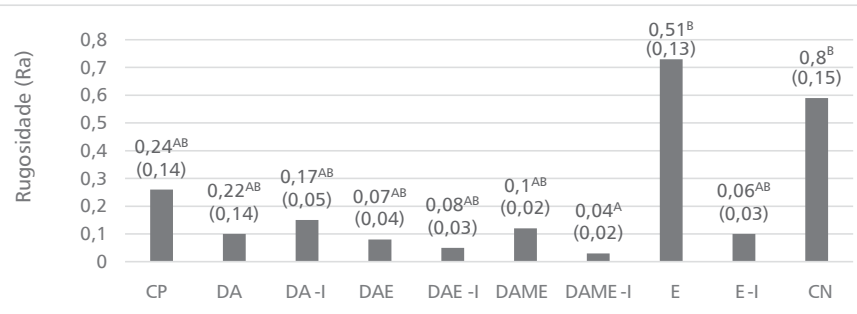

Figura 1 - Média (desvio-padrão) dos resultados rugosidade média (padrão Ra) para todos os grupos após acabamento e polimento e armazenamento em água $(\mathrm{n}=5)$. Letras diferentes mostram diferenças estatística para os testes ANOVA e Tukey $(\alpha=0,05)$. foram realizadas três vezes para cada espécime e foi adotada a média dos valores. Todas as medidas foram realizadas por um operador que desconhecia a que grupo pertenciam os espécimes e o espectrofotômetro foi calibrado a cada mensuração.

Para obtenção da variação de cor entre os períodos inicial, 7 e 14 dias foi calculada a diferença de cor ou $\Delta \mathrm{E}$, representado pela equação:

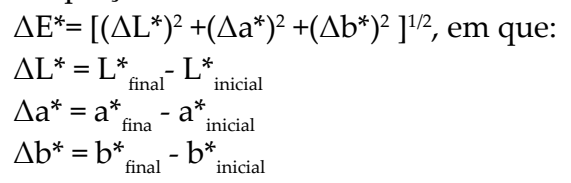

Morfologia de superfície (Microscopia Eletrônica de Varredura-MEV)

Um espécime de cada grupo foi selecionado aleatoriamente, para análise da morfologia de superfície após acabamento e polimento realizado pelos diferentes protocolos e armazenamento em água com ou sem imersão em café. Foi selecionada uma amostra de cada grupo que tenha sido armazenada em água, e uma com imersão em café, totalizando 20 amostras. A MEV foi realizada através de Microscópio Eletrônico de Varredura (JEOL JSM IT300LV, Akishima, Tóquio, Japão) em aumentos padronizados.

\section{Análise estatística dos dados}

Os dados coletados de rugosidade e cor foram submetidos aos testes de Kolmogorov-Smirnov e Shapiro-Wilk para verificar a normalidade de distribuição. Posteriormente, devido a distribuição paramétrica, para comparação entre todos os grupos foram realizados os testes Anova e Tukey. Para comparação entre os diferentes protocolos a seco ou sob irrigação foi utilizado o Teste T. Todos os testes foram realizados ao nível de significância de 5\% no programa SPSS20.0 (IBM, Chicago, EUA).

\section{RESULTADOS}

\section{Rugosidade superficial}

Os resultados de rugosidade superficial estão dispostos nas Figuras 1, 2, 3 e 4. A Figura 1 mostra todos os grupos que foram armazenados em água. Os grupos $\mathrm{E}$ e $\mathrm{CN}$ apresentaram os maiores valores de rugosidade, sendo estatisticamente semelhantes a todos os outros grupos $(\mathrm{p}>0,05)$, exceto DAME-I que apresentou os menores valores de rugosidade $(p<0,05)$.

A Figura 2 ilustra os resultados de rugosidade para os grupos com imersão em café. Os grupos E e CN também apresentaram os maiores valores de rugosidade sendo diferentes estatisticamente

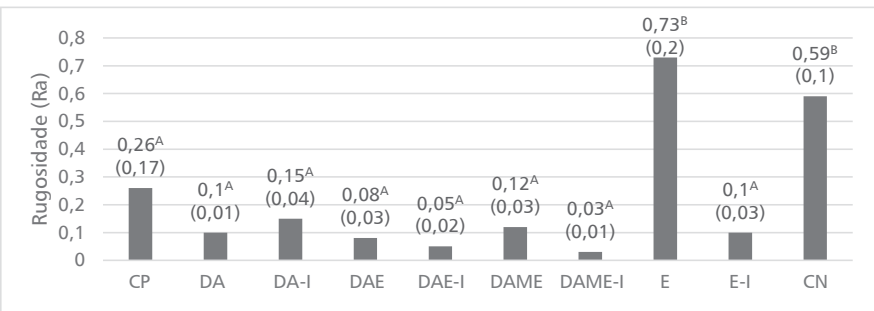

Figura 2 - Média (desvio-padrão) dos resultados rugosidade média (padrão Ra) para todos os grupos após acabamento e polimento e imersão em café $(\mathrm{n}=5)$. Letras diferentes mostram diferenças estatística para os testes ANOVA e Tukey $(\alpha=0,05)$. 
de todos os outros grupos $(\mathrm{p}<0,05)$, que não apresentaram diferença estatística $(p>0,05)$.

A Figura 3 ilustra o resultado do Teste T comparando o acabamento e polimento a seco ou com irrigação para cada grupo armazenado em água. Para os protocolos DA e DAE não houve diferença estatística entre o acabamento e polimento a seco ou com irrigação $(p>0,05)$. Para os protocolos DAME e E, o acabamento e polimento com irrigação promoveu valores estatisticamente menores de rugosidade em relação ao acabamento e polimento a seco após armazenamento em água $(\mathrm{p}<0,05)$.

A Figura 4 apresenta o resultado do Teste $\mathrm{T}$ comparando o acabamento e polimento a seco ou com irrigação para cada grupo avaliado com imersão em café. Para os protocolos DA e DAE não houve diferença estatística entre o acabamento e polimento a seco ou com irrigação $(p>0,05)$. Para os protocolos DAME e E, o acabamento e polimento com irrigação promoveu menores valores de rugosidade em relação ao acabamento e polimento a $\operatorname{seco}(\mathrm{p}<0,05)$.

\section{Estabilidade de cor}

Os resultados de variação de cor para os diferentes grupos e diferentes protocolos de armazenamento estão dispostos nas Tabelas 3, 4 e 5. Na Tabela 3 é possível observar o efeito dos permeantes
Tabela 3 - Média (desvio-padrão) da variação de cor $(\Delta \mathrm{E})$ nos períodos 0 a 7 dias, 8 a 14 dias e 0 a 14 dias para os diferentes grupos após armazenamento em água ou imersão em café.

\begin{tabular}{|c|c|c|c|c|c|c|}
\hline \multirow{2}{*}{ Grupos } & \multicolumn{2}{|c|}{$\Delta \mathrm{E}$ 0-7 dias } & \multicolumn{2}{|c|}{$\Delta \mathrm{E} 8-14$ dias } & \multicolumn{2}{|c|}{$\Delta \mathrm{E}$ 0-14 dias } \\
\hline & Água & Café & Água & Café & Água & Café \\
\hline$C P$ & $1,6(0,7)^{A}$ & $4,4(0,7)^{A}$ & $1,0(0,6)^{A B}$ & $3,7(1,1)^{\mathrm{ABC}}$ & $2,3(1,1)^{\mathrm{A}}$ & $7,7(1,7)^{A}$ \\
\hline DA & $1,5(0,6)^{A}$ & $7,8(0,7)^{\mathrm{BC}}$ & $1,1(0,5)^{\mathrm{AB}}$ & $3,1(0,4)^{A B}$ & $2,4(0,9)^{A}$ & $10,8(0,8)^{\mathrm{ABC}}$ \\
\hline DA-I & $2,4(1,1)^{A B}$ & $6,9(1,1)^{A B C}$ & $0,8(0,2)^{A B}$ & $5,0(1,9)^{B C}$ & $2,8(1,1)^{\mathrm{A}}$ & $11,4(1,2)^{\mathrm{BC}}$ \\
\hline DAE & $2,4(0,9)^{A B}$ & $8,0(1,6)^{B C}$ & $1,3(0,3)^{A B}$ & $3,5(0,4)^{\mathrm{ABC}}$ & $3,4(0,5)^{A}$ & $11,3(1,8)^{\mathrm{BC}}$ \\
\hline DAE-I & $2,0(0,3)^{A}$ & $5,1(0,9)^{A B}$ & $0,8(0,1)^{A B}$ & $4,1(0,7)^{A B C}$ & $2,5(0,5)^{A}$ & $9,1(1,2)^{A B}$ \\
\hline DAME & $1,9(0,4)^{\mathrm{A}}$ & $7,0(0,8)^{A B C}$ & $1,2(0,2)^{A B}$ & $3,9(0,5)^{\mathrm{ABC}}$ & $2,8(0,7)^{\mathrm{A}}$ & $10,8(1,1)^{\mathrm{ABC}}$ \\
\hline DAME-I & $1,8(0,3)^{A}$ & $7,6(1,4)^{B C}$ & $1,6(0,5)^{B}$ & $4,2(0,9)^{A B C}$ & $2,8(0,1)^{\mathrm{A}}$ & $11,8(0,8)^{\mathrm{BC}}$ \\
\hline$E$ & $2,2(0,4)^{A}$ & $9,7(1,6)^{c}$ & $0,9(0,4)^{A B}$ & $4,1(1,0)^{A B C}$ & $2,9(0,8)^{\mathrm{A}}$ & $13,9(2,4)^{c}$ \\
\hline E-I & $1,9(0,3)^{A}$ & $6,4(0,9)^{A B}$ & $0,5(0,3)^{A}$ & $2,4(0,5)^{\mathrm{A}}$ & $2,0(0,1)^{A}$ & $8,7(0,9)^{A B}$ \\
\hline $\mathrm{CN}$ & $3,9(1,6)^{\mathrm{B}}$ & $14,5(2,5)^{\mathrm{D}}$ & $0,9(0,3)^{A B}$ & $5,5(1,8)^{c}$ & $3,5(1,3)^{A}$ & $20(2,3)^{D}$ \\
\hline
\end{tabular}

*Letras diferentes na vertical mostram diferença estatística $(p<0,05)$ de acordo com os testes ANOVA e Tukey $(\alpha=0,05)$

Tabela 4 - Média (desvio-padrão) da variação de cor $(\Delta \mathrm{E})$ nos períodos 0 a 7 dias, 8 a 14 dias e 0 a 14 dias, para os diferentes protocolos de acabamento dos espécimes armazenados em água $(\mathrm{n}=5)$.

\begin{tabular}{|c|c|c|c|c|c|c|}
\hline \multicolumn{3}{|c|}{$\Delta \mathrm{E} 0-7$ dias } & \multicolumn{2}{|l|}{$\Delta \mathrm{E}$ 8-14 dias } & \multicolumn{2}{|c|}{$\Delta \mathrm{E}$ 0-14 dias } \\
\hline Protocolo Irrigação & A Seco & Com Irrigação & A Seco & Com Irrigação & A Seco & Com Irrigação \\
\hline DA & $1,5(0,6)^{A}$ & $2,7(1,1)^{A}$ & $1,1(0,5)^{A}$ & $0,8(0,2)^{A}$ & $2,4(0,9)^{A}$ & $2,8(1,1)^{\mathrm{A}}$ \\
\hline DAE & $2,4(0,9)^{A}$ & $2,0(0,3)^{A}$ & $1,3(0,3)^{A}$ & $0,8(0,1)^{A}$ & $3,4(0,5)^{\mathrm{B}}$ & $2,5(0,5)^{\mathrm{A}}$ \\
\hline DAME & $1,8(0,4)^{A}$ & $1,8(0,3)^{A}$ & $1,2(0,2)^{A}$ & $1,6(0,4)^{A}$ & $2,8(0,7)^{A}$ & $2,8(0,1)^{A}$ \\
\hline$E$ & $2,2(0,4)^{\mathrm{A}}$ & $1,9(0,3)^{\mathrm{A}}$ & $0,9(0,4)^{A}$ & $0,5(0,3)^{A}$ & $2,9(0,8)^{\mathrm{B}}$ & $2,0(0,1)^{\mathrm{A}}$ \\
\hline
\end{tabular}

* Letras diferentes mostram diferença estatística $(p<0,05)$ de acordo com o Teste T $(\alpha=0,05)$ para um mesmo protocolo de acabamento e polimento a seco ou sob irrigação (a seco x com irrigação).

Tabela 5 - Média (desvio-padrão) da variação de $(\Delta \mathrm{E})$ cor nos períodos 0 a 7 dias, 8 a 14 dias e 0 a 14 dias, para os diferentes protocolos de acabamento dos espécimes imersos em café $(\mathrm{n}=5)$.

\begin{tabular}{|c|c|c|c|c|c|c|}
\hline & $\Delta \mathrm{E}$ 0-7 dias & & $\Delta \mathrm{E}$ 8-14 dias & & $\Delta \mathrm{E} \mathrm{0-14} \mathrm{dia}$ & \\
\hline Protocolo Irrigação & À Seco & Com Irrigação & À Seco & Com Irrigação & À Seco & Com Irrigação \\
\hline DA & $7,8(0,7)^{A}$ & $6,9(0,9)^{A}$ & $3,1(0,4)^{\mathrm{A}}$ & $5,0(1,9)^{A}$ & $10,8(0,8)^{A}$ & $11,4(1,2)^{A}$ \\
\hline DAE & $8,0(1,6)^{B}$ & $5,1(0,9)^{A}$ & $3,5(0,3)^{A}$ & $4,1(0,7)^{A}$ & $11,3(1,8)^{A}$ & $9,1(1,2)^{A}$ \\
\hline DAME & $7,0(0,8)^{\mathrm{A}}$ & $7,6(1,4)^{A}$ & $3,9(0,5)^{A}$ & $4,2(0,9)^{A}$ & $10,8(1,1)^{A}$ & $11,8(3,1)^{\mathrm{A}}$ \\
\hline$E$ & $9,7(1,6)^{\mathrm{B}}$ & $6,4(0,9)^{A}$ & $4,1(1,0)^{\mathrm{B}}$ & $2,4(0,5)^{A}$ & $13,9(2,4)^{\mathrm{B}}$ & $8,7(0,9)^{\mathrm{A}}$ \\
\hline
\end{tabular}

* Letras diferentes mostram diferença estatística $(p<0,05)$ de acordo com o Teste T $(\alpha=0,05)$ para um mesmo protocolo de acabamento e polimento a seco ou com irrigação (a seco x com irrigação).

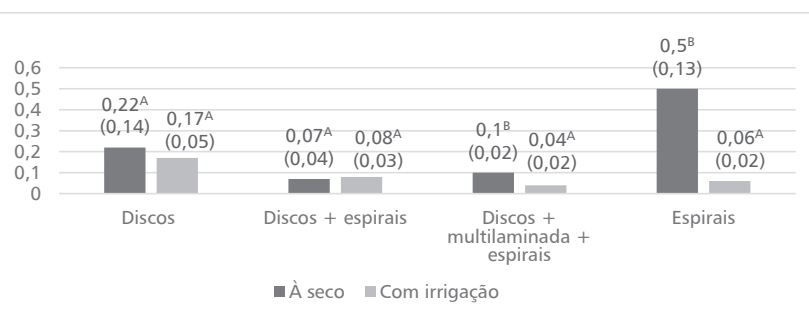

Figura 3 - Média (desvio-padrão) dos resultados rugosidade média (padrão Ra) para os diferentes protocolos de acabamento apos armazenamento em água $(n=5)$. Letras diferentes mostram diferença estatística $(\mathrm{p}<0,05)$ para o Teste $\mathrm{T}(\alpha=0,05)$ em um mesmo protocolo de acabamento e polimento à seco ou com irrigação.

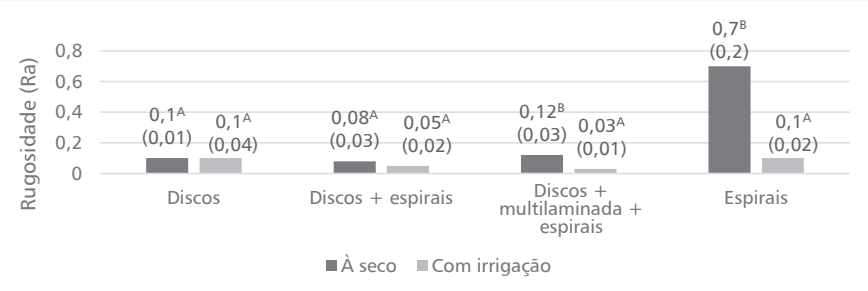

Figura 4 - Média (desvio-padrão) dos resultados rugosidade média (padrão Ra) para os diferentes protocolos de acabamento após imersão em café $(\mathrm{n}=5)$. Letras diferentes mostram diferença estatística $(\mathrm{p}<0,05)$ para o Teste $\mathrm{T}(\alpha=0,05)$ em um mesmo protocolo de acabamento e polimento à seco ou com irrigação. 
(água ou café) utilizados na variação de cor de cada um dos grupos. Nos primeiros 7 dias, após armazenamento em água ( $\triangle \mathrm{E}$ 0-7 dias), o grupo DA obteve a menor variação de cor $(\Delta E=1,5)$, sendo semelhante estatisticamente a todos os grupos $(p>0,05)$, exceto ao grupo $\mathrm{CN}$ (sem polimento) que obteve a maior variação de cor $(\Delta \mathrm{E}=3,9)$. Após imersões em café, o grupo $\mathrm{CP}$ (tira de poliéster) obteve a menor variação de cor $(\Delta E=4,4)$, sendo semelhante estatisticamente aos grupos DA-I, DAE-I, DAME e E-I. Os grupos $\mathrm{CN}$ e E apresentaram a maior variação de cor. No período de 8 a 14 dias, após armazenamento em água, o grupo DAME-I, apresentou a maior variação de cor $(\Delta E=1,6)$, sem diferença estatística dos demais grupos $(p>0,05)$, exceto E-I que teve a menor variação de cor $(p<0,05)$. Após imersão em café, o grupo $\mathrm{CN}$ apresentou a maior variação de cor sendo semelhante apenas ao grupo DA-I, e os outros grupos foram semelhantes entre si. Após os 14 dias de armazenamento em água a variação de cor de todos os grupos foi semelhante estatisticamente ( $p>0,05)$. Em relação aos espécimes que foram imersos em café, após os 14 dias, o grupo CN apresentou a maior variação de cor $(\Delta E=20)$, sendo seguido pelo grupo $\mathrm{E}$, sendo estes, diferentes estatisticamente $(\mathrm{p}<0,05)$. O grupo $\mathrm{CP}$ apresentou a menor variação de cor $(\Delta E=7,7)$, sendo semelhante aos grupos DA, DAE-I, DAME e E-I ( $>00,05)$.

A Tabela 4 representa os resultados após Teste $\mathrm{T}$, comparando o acabamento e polimento a seco ou com irrigação de cada protocolo quando armazenados somente em água. Nos primeiros 7 dias e no período entre 8 e 14 dias de armazenamento em água não houveram diferenças estatísticas entre o acabamento a seco e com irrigação para nenhum dos protocolos utilizados $(p>0,05)$. Após os 14 dias, os protocolos DAE e E mostraram estatisticamente menor variação de cor quando o acabamento e polimento foi realizado com irrigação $(p<0,05)$, para os outros protocolos não houve diferença estatística $(\mathrm{p}>0,05)$.

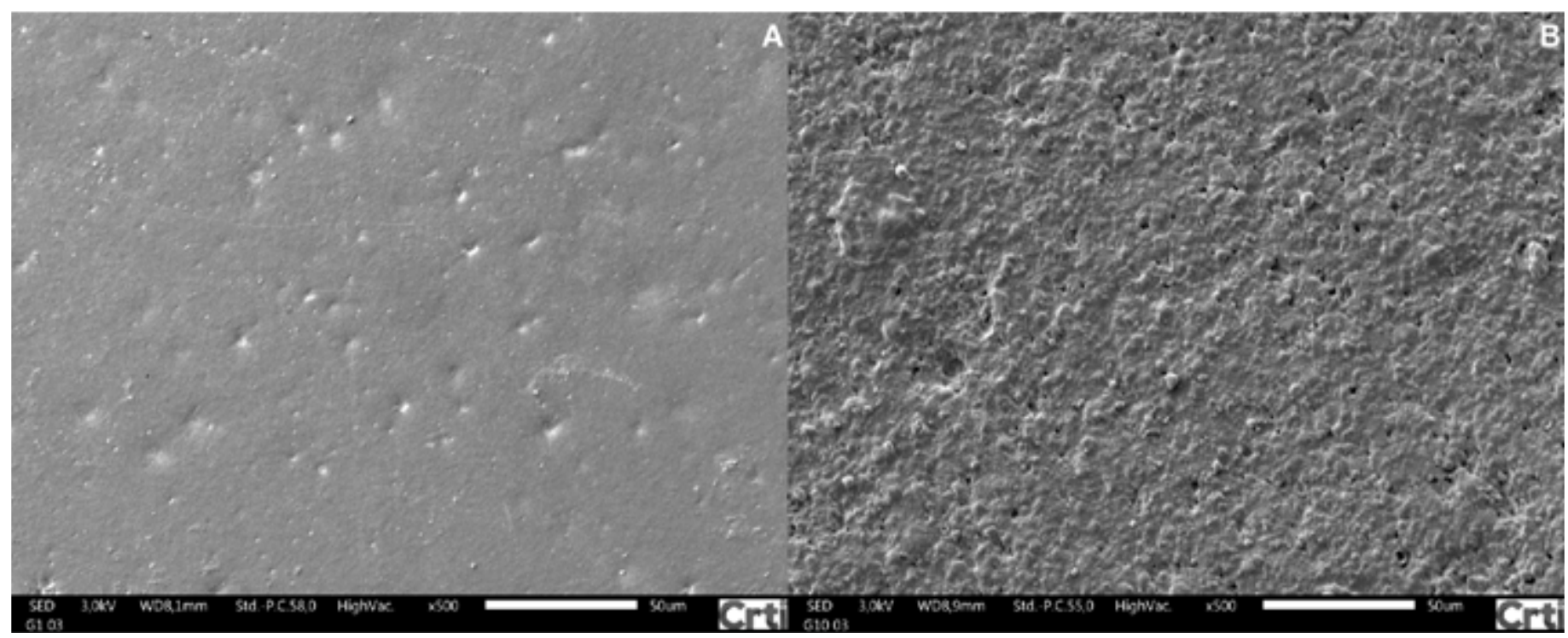

Figura 5 - Microscopia eletrônica de varredura mostrando a morfologia de superfície dos espécimes. (A) 500x Controle positivo (CP). (B) 500x Controle negativo (CN).

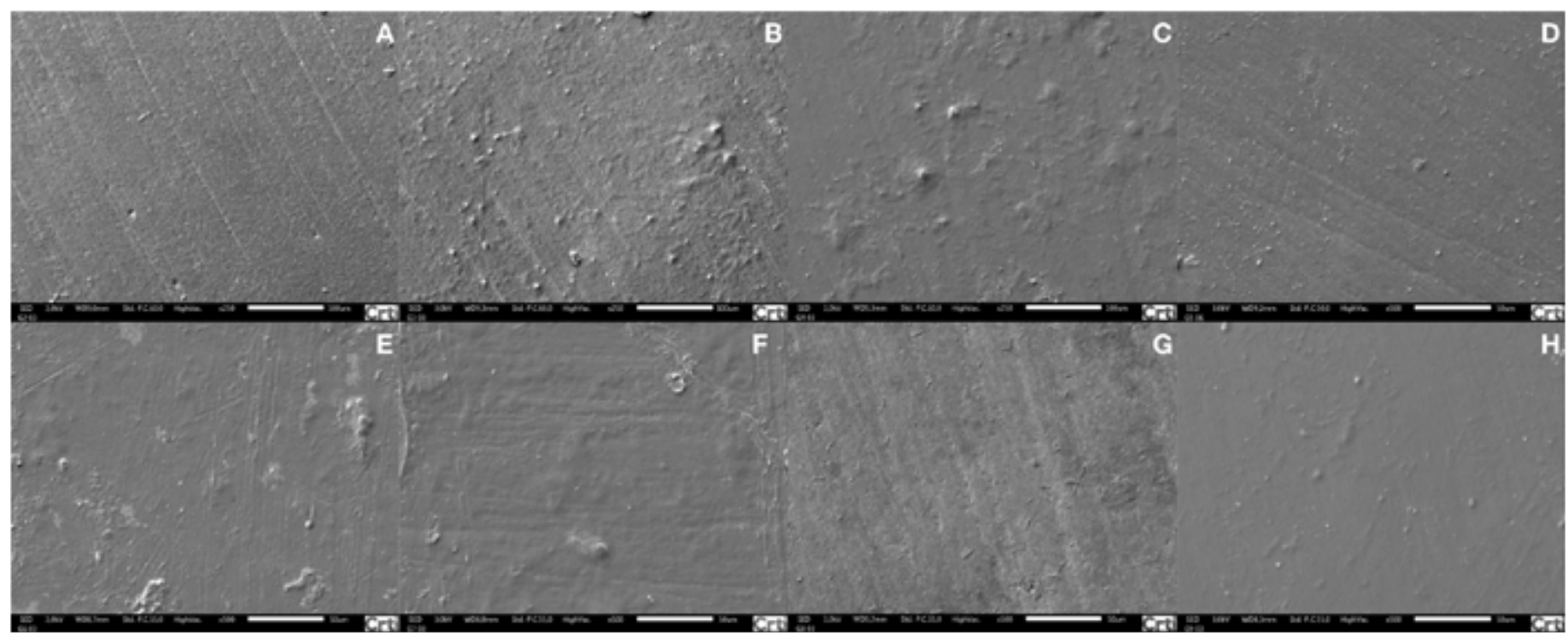

Figura 6 - Microscopia eletrônica de varredura mostrando a morfologia de superfície dos espécimes após os diferentes protocolos de acabamento e polimento. (A) 500 x Discos a seco. (B). Discos com irrigação. (C) $500 x$ Discos + espirais a seco. (D) $500 \times$ Discos + espirais com irrigação. (E) $500 \times$ Discos + multilaminada + espirais a seco. (F) Discos + multilaminda + espirais com irrigação. (G) 500x Espirais a seco. (H) 500x Espirais com irrigação. 
A Tabela 5 representa os resultados após Teste $\mathrm{T}$, comparando o acabamento e polimento a seco ou com irrigação de cada protocolo quando imersos em café. Nos primeiros 7 dias, os protocolos DAE e E mostraram estatisticamente menor variação de cor quando o acabamento e polimento foi realizado com irrigação $(p<0,05)$, para os outros protocolos não houve diferença estatística $(p>0,05)$. No período entre 8 e 14 dias de imersão em café, não houveram diferenças estatísticas entre o acabamento a seco e com irrigação para nenhum dos protocolos utilizados ( $\mathrm{p}>0,05)$. Após os 14 dias, os protocolos DAE e E mostraram estatisticamente menor variação de cor quando o acabamento e polimento foi realizado com irrigação $(p<0,05)$; para os outros materiais, não houve diferença estatística $(p>0,05)$.

\section{Morfologia de superfície (MEV)}

Os resultados observados em MEV estão apresentados nas Figuras 5 e 6 . Na Figura 5 é possível observar que apesar de o grupo $\mathrm{CP}$ (controle positivo) apresentar a superfície lisa, foi observada a presença de bolhas (Figura 5A). Na mesma figura, observa-se que o grupo $\mathrm{CN}$ (controle negativo) que apresentou grande irregularidade na superfície (Figura 5B).

Comparando-se os protocolos de acabamento e polimento na Figura 6, o uso de espirais com irrigação promoveu visualmente a maior lisura de superfície em relação aos outros grupos. Comparando o uso de espirais a seco e com irrigação é possível observar diferenças significativas no padrão de superfície obtido.

\section{DISCUSSÃO}

Este estudo avaliou a influência do protocolo de acabamento e polimento realizado com ou sem irrigação na rugosidade, variação de cor e morfologia de superfície de uma resina composta nanoparticulada após armazenamento em água com ou sem imersão em café. As hipóteses nulas de que o uso da irrigação não influenciaria a variação de cor, a rugosidade superficial e a morfologia de superfície e de que o protocolo de acabamento e polimento utilizado não influenciaria nas propriedades avaliadas foram rejeitadas. $\mathrm{O}$ uso da irrigação foi significativo, principalmente quando o polimento foi realizado com discos, ponta multi-laminada e espirais ou somente com espirais; e os diferentes protocolos de acabamento e polimento influenciaram na estabilidade de cor, rugosidade superficial e morfologia de superfície da resina composta.

Poucos estudos sobre o uso da irrigação durante o acabamento e polimento estão disponíveis na literatura. Em 2017, Nasoohi, Hoorizad e Tabatabaei ${ }^{15}$ avaliaram a influência da irrigação durante o uso de discos abrasivos (Sof-Lex Pop-On, 3M ESPE, EUA) na rugosidade superficial e microdureza de resinas compostas, e mostraram que o uso da irrigação promoveu menor rugosidade de superfície na resina composta. No presente estudo, o uso de discos abrasivos com e sem irrigação foi semelhante. Entretanto, para protocolos como DAME e E, o uso da água promoveu menor rugosidade de superfície na resina composta utilizada. Os protocolos DAE e E promoveram menor variação de cor quando o polimento foi realizado com irrigação. No polimento a seco, partículas abrasivas dos materiais de polimento podem ser impregnadas na superfície da resina composta, o que pode diminuir brilho e lisura de superfície, além disso, o superaquecimento durante o polimento a seco pode favorecer o deslocamento de partículas de carga, favorecendo o aumento da rugosidade de superfície ${ }^{14-16}$.

A rugosidade superficial é um dos parâmetros mais utilizados para a avaliar a qualidade de superfície dos materiais restauradores. Já que, menores valores de rugosidade remetem a menor manchamento da superfície e menor acúmulo de placa. Estudos mostram que valores de rugosidade de superfície acima de $0,2 \mu \mathrm{m}$ podem aumentar o acúmulo de placa e o risco de problemas periodontais ${ }^{17}$. Um estudo clínico mostrou que um valor de rugosidade de $0,3 \mu \mathrm{m}$ pode ser perceptível ao paciente ${ }^{18}$. No presente estudo, o protocolo com espirais a seco apresentou a maior média de rugosidade, sendo semelhante ao grupo em que nenhum polimento foi realizado, promovendo uma superfície inaceitável clinicamente. Um alto brilho e lisura de superfície são obtidos quando a resina composta é sobreposta com uma tira de poliéster antes da fotoativação ${ }^{19}$, porém este protocolo se torna inviável clinicamente, devido a necessidade do uso de brocas e discos para ajuste oclusal e obtenção de formas anatômicas adequadas para a restauração. No presente estudo, o uso da tira de poliéster teve resultado semelhante aos demais protocolos de polimento. O protocolo mais eficiente para a redução da rugosidade foi o que envolveu discos abrasivos, broca multi-laminada e espirais, porém, foram estatisticamente semelhantes aos demais.

Determinar os materiais e técnicas mais apropriados para acabamento e polimento, que alcançam um bom resultado de brilho e lisura superficial com menor quantidade de passos é um fator importante ${ }^{12,20}$. O uso de um protocolo com passos reduzidos, tal como, espirais com irrigação, se mostra tão efetivo quanto protocolos com maior quantidade de passos. Kemaloglu, Karacolak e Turkun, em 2016, mostraram de forma similar que sistemas simplificados, como as borrachas espirais criam uma superfície similar a um sistema de discos com várias granulações (Super Snap, Shofu, Japão). O sistema de borrachas espirais impregnadas com grânulos de óxido de alumínio e diamante (Sof-Lex Espiral, 3M ESPE, EUA) foi lançado com a intenção de reduzir os passos clínicos durante o acabamento e polimento. Seu bom desempenho pode estar associado a sua geometria e flexibilidade que facilita o contato com todas as superfícies da restauração, minimizando a formação de calor e a pressão indesejada ${ }^{21,22}$. As imagens de MEV mostram a maior lisura obtida pelo grupo E-I e o bom desempenho das borrachas espirais corrobora com outros estudos ${ }^{12,22}$. Entretanto, para o recontorno de restaurações, remoção de excessos e personalização morfológica, instrumentos abrasivos como discos e brocas são utilizados ${ }^{23,24}$, o que implica na necessidade da associação entre brocas, discos e borrachas para a obtenção do melhor resultado possível, neste caso o protocolo DAME se mostra eficiente.

A mudança de cor ao longo do tempo representa um problema para restaurações de resina composta e depende de vários fatores, tais como, ingestão de corantes, da resina composta utilizada e a rugosidade da superfície polida ${ }^{25}$; assim como manutenção precária de higiene, hábitos pigmentares, técnica restauradora e qualidade do processo de polimerização. Estudos ${ }^{11,23}$ mostram que a técnica de acabamento e polimento influencia na estabilidade de cor de resinas compostas. Nos primeiros 7 dias, os espécimes que não passaram por acabamento e polimento sofreram variação de cor maior que os demais grupos, entretanto, após 14 dias de 
armazenamento em água não houve diferença significativa entre os grupos, e os grupos CN e DAE apresentaram maior variação de cor, sendo esta com resultado $\Delta$ maior que $3,3^{26}$ que se representa inaceitável clinicamente. Para os espécimes que foram imersos em café diariamente uma maior variação de cor foi observada, já que após os 14 dias, todos os grupos apresentaram variação de cor superior a 3,3, sendo considerada clinicamente inaceitável. Os grupos CN e E apresentaram a maior variação de cor após os 14 dias e os grupos CP e E-I apresentaram a menor variação de cor. Apesar disso E-I foi semelhante aos demais protocolos, um fato que pode ser explicado pelo uso de uma única resina composta no estudo. Beltrami (2018) ${ }^{11}$ mostrou que resinas nanoparticuladas apresentam melhor capacidade de polimento em relação a outros tipos de compósito e consequentemente menores diferenças entre vários tipos de materiais de polimento. Principalmente para os grupos DAE e E o uso da irrigação durante o acabamento e polimento foi significativo para reduzir a variação de cor, mostrando mais uma vez a importância do uso da irrigação principalmente quando as borrachas espirais são utilizadas.

As limitações desse estudo consistem no uso de somente um sistema de resina composta. Clinicamente, no ambiente oral, as margens da restauração estão mais propensas ao acúmulo de placa e descoloração. Estudos futuros, preferencialmente clínicos, e com um acompanhamento a longo prazo, são necessários para verificar o comportamento das restaurações frente a estes protocolos de acabamento e polimento.

\section{CONCLUSÃO}

Diante das limitações desse estudo é possível concluir que:

- Diferentes protocolos de acabamento e polimento influenciam na rugosidade superficial, variação de cor e morfologia de superfície de resinas compostas;

- O uso da irrigação foi significativo quando os protocolos utilizados foram discos abrasivos + multilaminada + espirais, e quando somente os espirais foram utilizados;

- As borrachas espirais apresentaram os menores valores de rugosidade e variação de cor e maior lisura de superfície quando utilizados com irrigação.

\section{REFERÊNCIAS}

01. Frese C, Schiller P, Staehle HJ, Wolff D. Recontouring teeth and closing diastemas with direct composite buildups: a 5-year followup. J Dent 2003;41:979-985.

02. de Moraes RR, Goncalves L de S, Lancellotti AC, Consani S, CorrerSobrinho L, Sinhoreti M. Nanohybrid resin composites: Nanofiller loaded materials or traditional microhybrid resins? Operative Dentistry 2009;34(5):551-557.

03. Palaniappan S, Bharadwaj D, Mattar DL, Peumans M, Van Meerbeek $\mathrm{B}$, \& Lambrechts P. Three-year randomized clinical trial to evaluate the clinical performance and wear of a nanocomposite versus a hybrid composite Dent Mat 2009;25(11) 1302-1314.

04. de Andrade AK, Duarte RM, Guedes Lima SJ, Passos TA, Lima KC, \& Montes MA. Nanohybrid versus nanofill composite in class I cavities: Margin analysis after 12 months Microscopy Research and Technique 2011;74(1) 23-27

05. Alzraikat H, Burrow MF, Maghaireh GA, Taha NA. Nanofilled resin composite properties and clinical performance: a review. Oper Dent 2018;43(4):173-190.
06. Jefferies SR. Abrasive finishing and polishing in restorative dentistry: A state-of-the-art review. Dent Clin North Am 2007;51:379-97.

07. Avsar A, Yuzbasioglu E, Sarac D. The effect of finishing and polishing techniques on the surface roughness and the color of nanocomposite resin restorative materials. Adv Clin Exp Med 2015; 24(5):881-890

08. Aytac F, Karaaslan ES, Agaccioglu M, Tastan E, Buldur M, Kuyucu E. Effects of Novel Finishing and Polishing Systems on Surface Roughness and Morphology of Nanocomposites. J Esthet Restor Dent. 2016 Jul;28(4):247-61.

09. Paravina RD, Roeder L, Lu H, Vogel K, Powers JM. Effect of finishing and polishing procedures on surface roughness, gloss and color of resin-based composites. Am J Dent 2004;17:262-6.

10. Carneiro PMA, Ramos TM, de Azevedo CS, de Lima E, de Souza SHJ, et al. Influence of Finishing and Polishing Techniques and Abrasion on Transmittance and Roughness of Composite Resins. Oper Dent 2016;41(6);634-641.

11. Beltrami R, Ceci M, De Pani G, Vialba L, Federico R, Poggio C et al. Effect of different surface finishing/polishing procedures on color stability of esthetic restorative materials: A spectrophotometric evaluation. Eur J Dent 2018;12:49-56.

12. Kemaloglu H, Karacolak G, Turkun LS. Can Reduced-Step Polishers Be as Effective as Multiple-Step Polishers in Enhancing Surface Smoothness? J Esthet Restor Dent. 2017 Feb;29(1):31-40.

13. Kamined RR, Penumatsa NV, Priya T, Baroudi K. The influence of finishing/polishing time and cooling system on surface roughness and microhardness of two different types of composite resin restorations. J Int Soc Prev Community Dent. 2014 Dec;4(Suppl 2):S99-S104.

14. Dodge WW, Dale RA, Cooley RL, Duke ES. Comparison of wet and dry finishing of resin composites with aluminum oxide discs. Dent Mater. 1991 Jan;7(1):18-20.

15. Nasoohi N, Hoorizad M, Tababtabei SF. Effects of Wet and Dry Finishing and Polishing on Surface Roughness and Microhardness of Composite Resins. Journal of Dentistry (Tehran) 2017;14(2):69-75.

16. Lopes GC, Franke M, Maia HP. Effect of finishing time and techniques on marginal sealing ability of two composite restorative materials. J Prosthet Dent. 2002 Jul;88(1):32-6.

17. Bollen CM, Lambrechts P, Quirynen M. Comparison of surface roughness of oral hard materials to the threshold surface roughness for bacterial plaque retention: a review of the literature. Dent Mater 1997;13:258-69.

18. Jones CS, Billington RW, Pearson GJ. The in vivo perception of roughness of restorations. Br Dent J 2004;196: 42-5.

19. Can Say E, Yurdaguven H, Yaman BC, Ozer F. Surface roughness and morphology of resin composites polished with two-step polishing systems. Dent Mater J 2014;33(3): 332-42.

20. Turkun LS, Turkun M. The effect of one-step polishing system on the surface roughness of three esthetic resin composite materials. Oper Dent 2004;29(2):203-11.

21. da Costa JB, Goncalves F, Ferracane JL. Comparison of two-step versus four-step composite finishing/polishing disc systems: evaluation of a new two-step composite polishing disc system. Oper Dent 2011; 36: 205-212.

22. Pala K, Tekçe N, Tuncer S, Serim ME, Demirici M. Evaluation of the surface hardness, roughness, gloss and color of composites after different finishing/polishing treatments and thermocycling using a multitechnique approach. Dent Mater J 2016; 35(2): 278-289.

23. Kumari RV, Nagaraj H, Siddaraju K, Poluri RK. Evaluation of the effect of surface polishing, oral beverages and food colorants on 
color stability and surface roughness of nanocomposite resins. J Int Oral Health 2015;7:63-70.

24. Ferraris F, Conti A. Superficial roughness on composite surface, composite enamel and composite dentin junctions after different finishing and polishing procedures. Part I: Roughness after treatments with tungsten carbide vs. Diamond burs. Int J Esthet Dent 2014;9:70-89.
25. Soares-Geraldo D, Scaramucci T, Steagall W Jr., Braga SR, Sobral MA. Interaction between staining and degradation of a composite resin in contact with colored foods. Braz Oral Res 2011;25:369-75.

26. Barath VS, Faber FJ, Westland S, Niedermeier J. Spectrophotometric analysis of all-ceramic materials and their interaction with luting agents and different backgrounds. Adv Dent Res 2003;17:55-60.

\section{ABSTRACT}

Aim: to evaluate the surface roughness (SR), color stability (CE) and surface morphology (SM) of a nanofilled composite resin exposed to different finishing/ polishing protocols with or without irrigation. Material and methods: One hundred specimens were prepared and divided into 10 groups $(n=10)$ according to the finishing/polishing protocol: positive control; abrasive discs (AD); AD + spirals (ADS); AD + multi-laminated + spirals (ADMS); spirals (S); and none. Protocols with AD; ADS; ADMS and $S$ were performed to dry or with irrigation. After finishing/ polishing, the samples were stored in distilled water at $37^{\circ} \mathrm{C}$. For 14 days, five samples of each group were immersed in coffee for $5 \mathrm{~min} /$ day. The other samples have been stored in water. The CE of the samples was analyzed with Spectrophotometer $\left(\mathrm{CIEL}^{*} \mathrm{a}^{*} \mathrm{~b}^{*}\right)$ 0, 7 and 14 days after storage in water or coffee. The SR was analyzed with the Rugosimeter and the SM was analyzed with Scanning Electron Microscopy (SEM). ANOVA, Tukey and T-test were performed for comparisons between the groups $(\alpha=0,05)$. Results: Irrigation offered higher EC in the ADS and $S$ groups. The benefits of color variation are after 14 days. The use of dry spirals promoted the highest SR values, being lower in the ADMS group, which promoted less roughness and was similar to the other protocols. For ADMS and $S$ the irrigation was significant to decrease the roughness. Analysis by MEV, spirals with irrigation greater superficial smoothness. Conclusion: Irrigation during finishing/polishing influences the CS, SR and SM of composites. The finishing/polishing protocols with ADMS and $S$ with irrigation were more effective.

Key-words: polishing techniques; surfasse roughness; color variation; nanofilled composites.

\section{AGRADECIMENTOS}

Agradecemos ao Centro Regional Para o Densenvolvimento Tecnológico e Informação (CRTI) da Universidade Federal de Goiás, onde foram realizadas as análises de microscopia eletrônica de varredura deste trabalho, e a 3M ESPE pela doação de parte dos materiais utilizados neste estudo.

\section{AUTOR PARA CORRESPONDÊNCIA}

Rafael de Almeida Decurcio

Avenida Itália, 1184, 74325-110, Goiânia, GO

cientifico@abo-go.org.br 